\title{
Los posgrados y la deuda de la investigación científica en las universidades de la región occidental de Honduras
}

\author{
Alfonso Carranza Ramos, Jorge Humberto Orellana Peña*
}

\section{RESUMEN}

La región occidental de Honduras, es un territorio complejo en su configuración social, económica, ambiental, étnica, cultural e histórica; y además en permanente proceso de cambio y adaptación en su estructura y en la contradicción de sus fuerzas. Es una región con pobreza, vulnerabilidad, inequidad y exclusión social, con bajos indicadores de desarrollo humano. Este artículo presenta esos problemas que deben estudiarse desde los espacios académicos, especialmente de posgrado, para acercarse a la construcción de nuevos caminos que nos conduzcan a escenarios de desarrollo local, regional, humano, endógeno, multiétnico, pluricultural, horizontales y menos hegemónicos. Enfatizando en los valores del contexto regional y el rol trascendental que deben desempeñar las universidades en la producción de saberes mediante la investigación científica.

Se trata de completar y retomar ideas de aquellos elementos que proporcionen nuevas probabilidades para construir caminos donde toda la población tenga la oportunidad de participar, con sus experiencias, sueños, ideas y acciones más inclusivas que contribuyan a desarrollar potencialidades. Las universidades deben a través de la investigación estudiar la comprensión de esas realidades complejas como las del occidente de Honduras. Los pregrados deben ser el inicio para ese proceso de formación. Los posgrados por su parte, deberán entonces ser los espacios para profundizar, para entrar a los tejidos de sociedades como las poblaciones rurales de esta parte del país.

Palabras clave: Investigación, universidad, occidente, región, científica, conocimiento, posgrados.

\section{ABSTRACT}

Honduras' western region is a complex territory in all its settings: social, economic, environmental, ethnic, cultural and historical; besides its constant process of change and adaptation in regard to the contradiction of its forces. The region is characterized

\footnotetext{
* Universidad Nacional Autónoma de Honduras. Centro Universitario Regional de Occidente (CUROC). Coordinación Regional de Investigación Científica. jorellana4000@yahoo.es; alfonsocarranzar@yahoo.es
} 
by poverty, vulnerability, inequality and social exclusion, with low standards of human development. This article presents these problems which must be studied from the academy, especially in postgraduate spaces, in order to build new roads that may lead to local development scenarios. The emphasis on regional context values and the trascendental role that the universities holds must recover the production of knowledge by scientific research means.

The study tries to complete and take ideas from those elements that have supplied new probabilities to build new roads where all the population has the oportunity to participate with their own experiences, dreams, ideas, and more exclusive actions for the contribution of potential development. Universities must comprehend these realities through the contribution of research. The beginning process must start in the undergraduate programs. The postgraduate programs must be able to create spaces to deepen these studies much further.

Key words: Research, university, west region, scientific knowledge, postgraduate. 


\section{INTRODUCCIÓN}

La investigación científica en las universidades y en sus diferentes centros regionales, es un objetivo trascendental y una necesidad ingente que se debe emprender y fortalecer. La Universidad Nacional Autónoma de Honduras consciente de ese propósito, ha dedicado los últimos años, por lineamientos de la reforma, a promover mayores espacios en temas de investigación y publicaciones científicas. Sin embargo, esta cultura investigativa se ha convertido en una tarea pendiente en muchos centros universitarios públicos y privados del país. Hasta ahora el desempeño de los profesores en las universidades sigue siendo la docencia, dejando de lado otras tareas de relevancia como la vinculación con la sociedad y la investigación científica aplicada. La universidad y todo centro regional, debe reconocer la importancia de la generación de nuevos aprendizajes en grados y posgrados por su doble propósito; como espacio de formación y por su aporte a la generación de conocimiento y producción de ciencia.

Este artículo enfatiza en la cuota de responsabilidad del aspecto investigativo que tienen y deben satisfacer las universidades en el occidente de Honduras. Recalca la importancia de los posgrados, la generación de conocimiento y especialmente, hacia dónde deberían apuntar los procesos de investigación en una de las regiones más postergadas del país. También critica el hecho de que muchos proyectos de desarrollo han fracasado porque se han realizado desde una visión descendente y verticalista, como diría Octavio Paz, "no quieren cambiar, sino durar". Al mismo tiempo, la región ha sido el laboratorio más grande que sobre procesos de desarrollo haya existido en el país, la cual la convierte en un reto en sí misma; el desarrollo de organizaciones cooperativas en el occidente de Honduras, es quizá el área más importante de la economía social del país. El proceso de organización y reflexión desde las comunidades por las diferentes instituciones, es también otro de los grandes elementos a estudiar. La articulación en procesos de encadenamiento a los mercados de café de calidad, es un ejemplo más que debe profundizase para comprender sus dinámicas.

Las universidades públicas y el crecimiento de centros privados de educación superior aún tienen una deuda científica con los municipios de Honduras. Varias carreras de pregrado y algunas especializaciones de posgrado, se han implementado sin el conocimiento previo de la demanda profesional de la región. Esto implica que muchas intervenciones se han convertido simplemente en proyectos de supervivencia, a pesar de los cuantiosos fondos invertidos, especialmente de la cooperación solidaria internacional, occidente sigue hoy tan 
desigual como antes; manteniendo los mismos índices de inequidad y bajo desarrollo humano. Por lo tanto, sí la investigación es un componente esencial del desarrollo independientemente de los diferentes tipos, modelos y formas que existen, hacia ¿Dónde deben apuntar las investigaciones de posgrado? Consideramos que la academia debe formular conocimiento de nuevos comportamientos, de saberes holísticos, investigar la estructura del "mundo" local, la complementariedad en los procesos, porque no se puede plantear algo sin conocerlo, sin estudiarlo, indagar en la gente misma, en los pueblos, en los diferentes segmentos de la sociedad, desde la base de su propia estructura desarrollada en la cotidianidad de sus vidas. Es necesario investigar tecnologías, uso de recursos, economía, sincretismos; es decir, indagar, articular y construir saberes como parte esencial de una cultura dinámica, en permanente cambio, se trata de la búsqueda y de la construcción de una nueva epistemología desde una visión incluyente y principalmente, humana.

\section{EL CONTEXTO DE LAREGIÓN OCCIDENTAL DE HONDURAS}

La región occidental de Honduras se ubica al noreste de las repúblicas de Guatemala y El Salvador y al oeste de los departamentos de Santa Bárbara y Cortés, con una superficie de $9,100 \mathrm{~km}^{2}$ que representa el $8 \%$ del territorio nacional, en donde viven 754,909 personas (PNUD, 2009, pp. 417-435). Su topografía es muy irregular por ubicarse en la zona de la cordillera central y del sur, de donde se desprenden las sierras del Gallinero, Merendón, Celaque y Puca Opalaca, cuyas altitudes máximas oscilan entre $1400 \mathrm{~m} / \mathrm{nm}$ y $2385 \mathrm{~m} / \mathrm{nm}$. El área más alta de la región está situada en la montaña de Celaque, es la más antigua del país geológicamente hablando, y es considerada la más agreste, con apenas un $25 \%$ de territorio relativamente plano. Sus temperaturas oscilan entre los 11 y 33 grados centígrados, con precipitaciones que van desde los 1300 a 2200 mm/año. Los sistemas de pequeñas lagunas, lagunetas, aguas termales, bosques nublados, su red de ríos con todos sus sistemas de cuencas, hacen de esta región la más abundante en cuanto a captación de agua en el país, es aquí, donde se originan los más importantes afluentes de los ríos, Ulúa y Chamelecón. (Carranza, 2012, pp. 2426).

\section{Economía y Producción}

La economía del occidente del país, ha estado marcada por condiciones que se remontan a los tiempos de la colonia. Antes de la llegada de los españoles a esta zona, existieron rutas comerciales de larga distancia que se consolidaron durante el primer milenio de la era cristiana. Los Nahuas fue el grupo indígena que tuvo mayor 
influencia en la cultura y en el comercio en el occidente del país, "se establecieron en la costa del Caribe, desde donde expandieron el comercio a los pueblos del interior de Honduras, este acercamiento de comercio con los pueblos indígenas permitió la culturización de los nahuas sobre los pueblos" (Orellana, 2010, p. 42). Estas rutas siguieron utilizándose durante la "Colonia" para la comercialización del añil, principal rubro de exportación durante el siglo XVII (Ventura, 2009, p. 105). Al comenzar el periodo de independencia en el país, abandonada la producción del añil, empezó en la región occidental el cultivo del tabaco, actividad que se había iniciado a finales del periodo colonial, éste le dio un auge económico a la misma. El cultivo del tabaco en el occidente de Honduras, según Castañeda, se inició en Quezailica, departamento de Copán (Castañeda, 1946, p. 8). Varios cosecheros de esta aldea se trasladaron a los Llanos de Santa Rosa para continuar el cultivo de esta planta y dieron posteriormente vida a lo que ahora es Santa Rosa de Copán.

En la actualidad, por el predominio de una población rural, el occidente se caracteriza por un comportamiento económico basado en actividades económicas agrícolas. Está socio economía está fundamentada en tres pilares: La generada desde las pequeñas fincas de café (38\% de la producción de Honduras), las remesas enviadas por los hombres y mujeres que emigran a otros países y la pequeña empresa de carácter familiar, informal en su mayoría. Los pequeños y medianos productores del sector rural se desenvuelven en condiciones de producción de subsistencia y un acceso al mercado que no les permite solvencia económica para cubrir con sus necesidades básicas. A esto se suma las dificultades de acceso al crédito del sistema bancario nacional, en donde existe una brecha crediticia para rubros como el café, banano, hortalizas, granos básicos, entre otros. En vez de esto, se prefiere financiar importaciones por "no estar sujetas a los riesgos propios de la siembra y cosecha en el territorio nacional" (Perdomo, R., Díaz, M., 2012, p. 72). La concentración de las tierras fértiles y planas en pocas manos, se constituyó desde tiempos antiguos en un verdadero problema para los pequeños productores de la región. "El estado español distribuyó su usufructo entre los colonos, la iglesia entre los colonos, la iglesia y los indígenas (tierras comunales y ejidales)" (Varela, 2011, p. 146). Esto significó que las mejores tierras del occidente del país, quedaran en propiedad de los colonos y sus descendientes (criollos).

Desde mediados del siglo XX, diferentes estudios han mostrado que occidente sigue siendo una de las regiones con mayor rezago, aislamiento y pobreza, aunque algunos municipios con leves repuntes que otros. Los departamentos de Copán, Lempira y Ocotepeque se caracterizan por su desarticulación y desintegración con respecto al gobierno central y al resto del país. Al ser muy tardía la conformación de una estructura agroexportadora y agroindustrial, implica, entre otros fenómenos, la 
persistencia de altos niveles de pobreza e inequidad socioeconómica. (PLATSUNAH, 2005, p. 33). A pesar de ello, el occidente de Honduras presenta un mosaico de diversidad ambiental, suelos heterogéneos, ricos en las pocas hondonadas suaves y vegas; pobres en las muchas laderas, en donde las poblaciones pobres a lo largo del tiempo, han sido ubicadas, con su propia dinámica (Carranza, 2004, p. 13). Otro factor en la economía según el Postgrado Latinoamericano en Trabajo Social, es el predominio de "estructuras sociales de hacienda tradicional relacionada a la economía de frontera con un relativo desarrollo de una economía agropecuaria, una amplia población campesina con grandes carencias en las condiciones de vida y desarrollo relativo de un sector artesanal" (PLATS-UNAH, 2005. Op. cit., p. 52). Los productos que sobresalen actualmente son: café, tabaco, granos básicos, verduras, legumbres, frutas, caña de azúcar, ganado, explotación de madera y venta de servicios; esta producción se ve obstaculizada por los altos costos de los insumos, la débil comercialización y por la frágil infraestructura vial.

Con respecto a la producción del café, rubro que contribuye significativamente a la socio economía regional, en un reciente estudio realizado por el profesor Alfonso Carranza, sostiene que el café a occidente llegó entre los años de 1835 y 1850, un periodo relativamente corto, y se afirma que provino de Guatemala y El Salvador dada la propagación que existía en esos países. (Carranza, 2012. Op. cit., p. 60). El autor además menciona que la historia del café en esta zona se debe fundamentalmente a tres razones básicas: 1) a las circunstancias históricas propias, que a través de los tiempos en su suelo han ocurrido. 2); a sus condiciones agroecológicas, $y, 3$ ) a la cultura que sobre el café mismo y su gente a lo largo de los años ha ido estructurando. (Ibid, p. 14). Hoy el café en el occidente de Honduras, constituye la principal estrategia de vida y desarrollo para la población y la distribución de los beneficios alcanza a la mayoría de los participantes en la cadena de producción, algunos más que a otros. La principal razón de tal distribución es que todo su proceso se hace en su mayoría de forma manual. El estudio "Historia y Cultura del Café en el Occidente de Honduras", menciona datos interesantes acerca de las familias productoras. En esta región viven 24,925 familias productoras de café, que poseen 91,925 manzanas cultivadas, cuya contribución equivale al 38\% de la producción nacional con datos de cosecha 2010-2011 (Ibid. pp. 18-19).

Sus plantaciones han sido factor decisivo para aproximarse a mejores condiciones de vida, a una mejor distribución del ingreso, acercar oportunidades y posibilidades, a ser parte de ambientes naturales, definiendo los procesos de reinversión social y productiva. Pero estos beneficios, sin duda alguna, tienen que ver con una mejor repartición de las tierras. En promedio cada familia cultiva cuatro manzanas de 
café, de esta forma, la producción y el proceso mismo, no está condicionado ni concentrado en pocas manos, contrario a otros rubros de producción del resto del país.

\section{La Conquista, la Colonia y la Influencia Cultural en la Región Occidental de Honduras}

La historia del occidente de Honduras no nace con la aparición del proceso de conquista española, su historia se remonta a la época prehispánica en donde poblaciones como la gran civilización Maya escribieron en piedra sus memorias; durante el periodo de la colonia entre 1502 y 1821 se suscitaron una serie de cambios políticos, económicos y sociales que marcaron sus condiciones de vida (Zúñiga, 2000, p. 60-118). Durante la pacificación de esta región en 1536, dirigida por Alvarado, se produjo la despoblación de muchas comunidades en los alrededores de Gracias a Dios (hoy Lempira). Esta provincia comprendía, en los tiempos de la colonia, los departamentos de Copán, Ocotepeque, Lempira e Intibucá. En 1801 el Gobernador intendente Ramón de Anguiano reportó que la población de esta provincia tenía 20,000 habitantes ladinos y 20,000 indígenas distribuidos en 39 pueblos (Carranza, 2004. Op. cit., p. 54). El proceso de conquista y la aparición de la colonia y dominio español en la zona occidental, desplazó comunidades, cambiaron los patrones de vida, se apropiaron de los recursos, alteraron sus costumbres y destruyeron gran parte de su cultura tradicional.

La redistribución de tierras y reasentamientos de la población permitió las condiciones para la creación de las llamadas "Encomiendas" las cuales fueron otorgadas a varios españoles para su explotación y beneficio. Newson (2000) señala que: "Las encomiendas eran una institución inestable no sólo por el constante cambio de encomenderos, sino por la naturaleza misma de la institución" (p. 150). Asimismo, Orellana (2010) menciona que estos sistemas de reducción de indios fueron núcleos poblacionales en donde se reasentó la población con fines evangelizadores en la fe cristiana, "se dice que este tipo de organización data de 1531, en cada reducción de indios debía haber una iglesia o parroquia" (p. 45). Piraera, Maxaitique y Cascarón, Colohete, Caiquín, Erandique, Laganguera, entre otras, fueron encomiendas que generaron a la corona múltiples beneficios en la región, "se puede inferir que Piraera fue una encomienda muy productiva en el occidente del país" (Ventura, 2009. Op. cit, p. 82).

Barahona (2002) menciona que en el partido de Gracias a Dios existieron 48 reducciones, la población indígena se concentró en 39 pueblos (p. 185). En aquellos donde se concentraron los núcleos poblacionales se fundaron 35 "Curatos" que 
constituyeron el obispado de Honduras; una cantidad considerable se ubicó en el "Partido de Gracias a Dios", éste a su vez se distribuyó en parroquias (Leyva, 1991, p. 282-283). De la población indígena según Rivas (2000), sobreviven los Chortís; localizados en los departamentos de Copán y Ocotepeque con 3,500 habitantes. Lencas; ubicados en los departamentos de la Paz, Intibucá, Lempira y parte del departamento de Ocotepeque, con una población estimada en 90,000 habitantes. Los lencas con 612 comunidades, tienen un promedio de 130 habitantes por comunidad (p. 47-48).

\section{Condiciones de Desarrollo Humano y Pobreza}

Desde mediados del siglo XX, diferentes estudios han mostrado que occidente sigue siendo una de las regiones con mayor rezago, aislamiento y pobreza. En promedio el índice de desarrollo humano para 2007 en esta región osciló entre 0.600 y 0.699 ; sin embargo, en cada departamento existen varios municipios con precarias condiciones de vida. En los tres departamentos de la región el avance del desarrollo apenas es perceptible, con carácter heterogéneo y con desequilibrios muy marcados. La asistencia que el gobierno ha brindado a estos departamentos no ha sido sistemática, no han logrado reducir la brecha de inequidad y los programas y proyectos no han correspondido con el nivel de necesidades. Según datos del PNUD 2009 el índice de pobreza humana (IPH) para 2006 de los departamentos fue el siguiente:

\section{Índice de Pobreza Humana (2006)}

\begin{tabular}{|c|c|c|c|}
\hline Lempira & Copán & Ocotepeque & Promedio Nacional \\
\hline 22.8 & 21.3 & 17.8 & 13.5 \\
\hline
\end{tabular}

Fuente: Elaborado con datos del PNUD 2009

San Andrés, municipio del departamento de Lempira se encuentra con el índice de pobreza humana más alto (39.6). San Agustín en Copán con (32.6) y Dolores Merendón en Ocotepeque con (34.0) de IPH. A pesar de todas las necesidades de la región occidental, en ella existen una serie de condiciones y oportunidades para propiciar mejores derroteros.

Esta se ubica en el centro del área del desarrollo económico centroamericano, Guatemala y El Salvador y la costa norte hondureña, triángulo que se caracteriza por un alto dinamismo económico y tecnológico. Occidente posee una riqueza biótica, arqueológica y étnico-cultural. 


\section{LAS UNIVERSIDADES EN EL DESARROLLO DEL OCCIDENTE DE HONDURAS}

Las Universidades han sido consideradas centros o espacios donde se imparten y comparten conocimientos; donde se originan y se promueven debates, se analizan, se estudian las ideas, donde se innova, se plantean procesos, cambios y efectos; donde se discuten nuevas visiones. Se estudian los campos del conocimiento técnico-científico, social, económico, antropológico histórico, los estudios del desarrollo, la economía y otra serie de saberes y conocimientos importantes para las sociedades. El arte, literatura, filosofía, son también elementos del conocimiento encaminados hacia la formación. En Honduras existen 20 universidades, de las cuales 6 son públicas y 14 privadas. Al finalizar la década de los noventa en el país solo existían trece universidades, en 2007 se llegó a 20. Aunque el crecimiento de los centros privados ha sido notorio en las últimas décadas, la mayor cantidad de estudiantes sigue concentrado en las universidades públicas. Según el PNUD 2009, la cobertura neta de la educación superior casi se ha duplicado en el periodo comprendido entre 1990 y 2006 , pasando de $7.6 \%$ a $14.5 \%$ (p. 112).

Por otra parte, el Centro Interuniversitario de Desarrollo CINDA, revela en su informe "La Educación Superior en Iberoamérica 2011" que Honduras en 2010 tuvo un crecimiento en la matrícula de pregrado del $20 \%$ con relación al año 2005 . El mismo informe muestra que a nivel de posgrado, en el año 2005 las universidades públicas matricularon el $60 \%$ de la población estudiantil, de estos la UNAH atendió el $49 \%$, por su parte, las universidades privadas atendieron en el mismo año el $40 \%$. Para el año 2009, las universidades privadas atendieron el $68 \%$ de la población y el restante fue asumido por las universidades públicas (Duriez, M., Sándigo, C., 2011). Aunque hemos mejorado en términos de cobertura y equidad en los últimos años, es evidente que la calidad de la educación es todavía una tarea pendiente. Con el surgimiento cada vez más de universidades privadas, el número de egresados de carreras como derecho, ciencias sociales y educación comercial se ha incrementado. Paradójicamente en un país que predomina la actividad agrícola, la cantidad de graduados y la oferta en esta rama es muy limitada.

El estudio de CINDA, también revela que en la matrícula de los posgrados en 2009, la población se concentró en la Universidad Tecnológica Centroamericana y en la Universidad Católica de Honduras. Sin embargo, la matricula en el nivel de posgrado sigue siendo baja y menor aun en estudiantes de doctorado. En el occidente de Honduras tienen presencia tres universidades privadas y dos públicas, con disciplinas de aprendizajes parciales, con una demanda que está en función subjetiva en la esencia de servicio o clientelismo y no en la esencia de la necesidad. Esa fragmentación de los conocimientos como dice Morín, "no permite tener una 
visión del todo", lo cual muchas veces conduce inevitablemente a formar profesionales para un mundo que no existe. Al no realizarse diagnósticos con rigurosidad científica, las universidades a través de las diferentes carreras, satisfacen una demanda pero no una necesidad. El artículo 160 de la Constitución de la República, confiere a la Universidad Nacional Autónoma de Honduras la responsabilidad de desarrollar y organizar la educación superior en el país. "La Universidad Nacional Autónoma de Honduras es una institución autónoma del Estado, con personería Jurídica, goza de la exclusividad de organizar, dirigir y desarrollar la educación superior y profesional. Contribuirá a la investigación científica, humanística y tecnológica, a la difusión general de la cultura y al estudio de los problemas nacionales. Deberá programar su participación en la transformación de la sociedad hondureña". En este sentido, se necesita impulsar y coordinar mayores espacios desde la UNAH a través de su "Centro Regional Universitario" con las universidades de la región para liderar y fortalecer los temas de investigación, pertinencia y calidad de la educación superior.

A pesar de los obstáculos y limitaciones que enfrenta la UNAH, en sus objetivos esenciales de; Docencia, Investigación y Vinculación con la Sociedad, ha emprendido acciones en los últimos años para orientar la educación superior hacia la pertinencia, calidad, equidad, interdisciplinaridad e internacionalización. Los planteamientos en su "Modelo Educativo", buscan que sus docentes tengan competencias como: constructivista, agente de cambio, realista, didáctico, integrador, reflexivo y crítico, investigador, mediador, gestor y evaluador integral. Pero la mayoría de estos modelos se han quedado en los respectivos manuales, sin un seguimiento oportuno a las labores realizadas por los docentes. En su mayoría, los docentes desconocen el contenido esencial del nuevo modelo educativo de la UNAH. La mayor concentración del trabajo sigue siendo la docencia, dejando de lado el proceso de investigación y el fortalecimiento de aquellas iniciativas locales de la sociedad. En los temas de investigación, tanto de pregrado como posgrado, existe una mora o deuda significativa. La poca especialización del docente limita las posibilidades de búsqueda de conocimientos. La formación especializada que se ha recibido, más ha servido para buscar una reclasificación de categoría laboral, pero no necesariamente para apropiarse de la investigación científica.

En un estudio realizado por la doctora Margarita Oseguera acerca de las "Políticas de Investigación en las Universidades. El Caso de Honduras", menciona que la infraestructura para realizar investigaciones en la UNAH es limitada, a esto se suma la carencia de recursos económicos, muchas veces es desaprovechada esa oferta de financiamiento nacional e internacional por carecer de buenos gestores. Menciona también, que para la cantidad de docentes que laboran en las 
universidades, la producción científica es muy limitada; además el carácter burocrático del sistema deja de lado los aspectos eminentemente de gestión académica. Es imprescindible fortalecer la coordinación interinstitucional con diversos sectores de la sociedad, en el caso particular del Centro Regional Universitario de Occidente CUROC, en 2012 retomó el proceso de gestión de firma de cartas de entendimiento con organizaciones no gubernamentales (ONG) de la región, permitiendo un acercamiento de colaboración e investigación con estas entidades y de esta manera, aproximarnos a buscar posibles salidas a esas necesidades más ingentes y a la mayor comprensión de las comunidades del occidente, especialmente, a valorar nuestra capacidad de respuesta. Los campos de la investigación para los estudiantes de posgrado son interesantes; el cambio climático, la seguridad alimentaria, el deterioro de los ecosistemas, la pobreza, la marginación y exclusión social, la gestión del agua, las energías renovables, el diálogo intercultural, la salud, educación, entre otros.

\section{LOS POSGRADOS, EL CONOCIMIENTO Y LA DEUDA DE LA INVESTIGACIÓN CIENTÍFICA}

Se pensó que los conocimientos eran impulsados desde afuera; en el caso de nuestro país, que el saber procedía siempre de otros lugares, no de aquí; a los grados académicos se les denominó "títulos" en remembranza del uso de figuras sociales de origen elitista. La investigación científica, no ha profundizado en el porqué de los fenómenos y de los hechos, el con qué tiene que ver un suceso en particular, sus aproximaciones a la comprensión de las poli causalidades de las monumentales realidades sociales, sus orígenes históricos, la articulación en sus propios procesos dentro de una zona y con el resto de una región. No se ha estudiado la comprensión de viejos conocimientos y sus circunstancias, su profundidad y explicación, así como la generación de nuevas ciencias y nuevos aprendizajes en sus diferentes disciplinas científicas. Se ha dejado de lado los estudios acerca de las condiciones históricas y actuales, la estructura de la pobreza y sus causas, la marginalidad y exclusión, las nuevas ideas tecnológicas y la administración. Todo eso debe ser la Universidad, le correspondería ser la propuesta teórica actual vista desde la complejidad del tejido social, desde su multidimensionalidad, la incorporación de supuestos, de sus vacíos y sus discontinuidades; su indeterminismo, desde la proyección y la búsqueda de escenarios posibles.

La investigación debe ser una permanente construcción del conocimiento (trabajar, jugar, observar, discutir, debatir, construir, crear, escenificar, teorizar, probar, experimentar, pragmatizar). Sería pues, como dice Edgar Morín "un viaje que no se 
inicia con un método, se inicia con la búsqueda de un método". Al contrario, el pensamiento que durante siglos ha predominado son las premisas de los conceptos hegemónicos de la Europa occidental y sus herederos. A las formas de imposición y esclavitud se le interpretó como "civilizarnos" y a la imposición de su religión se le llamó "cristianizarnos". La religión, las leyes y la educación oficial impartida se encargaron posteriormente de reproducir, legitimar y justificar el nuevo sistema. Sus valores, visiones y premisas fueron desde siempre, suyas; hasta que de las contradicciones surgidas de su propio sistema, se dividió a sí mismo, fracturándose en un evento conocido como "independencia" que la religiosidad cívico ideológica elevó al nivel de efemérides. Luego, las estructuras de la desigualdad social creada por el gobierno colonial, fue continuada por sus herederos, los procesos de despojo siguieron hasta nuestros días. Octavio Paz (2008 p. 209) diría: "se esconde bajo una máscara... del mismo modo que la nación".

Para los siglos XIX y XX el pensamiento fundamentado en el iluminismo, positivismo, el realismo clásico basado en sus postulados como el determinismo, la causalidad, la lógica lineal, la objetividad, la universalidad de caracteres matemáticos de origen cartesiano, el mecanicismo newtoniano, el racionalismo, las visiones reduccionistas de la ciencia; han predominado en su configuración conceptual y ha sido parte esencial de la visión de las universidades. El mundo solo ha sido posible verlo desde sus análisis, desde la separación de sus partes y el estudio de ellas. David Bohm (1996 p. 11) en su obra Sobre el Diálogo menciona: "Los procesos genéricos del pensamiento humano, separan las cosas que en realidad, no se hallan separadas y nos llevan a percibir un mundo de manera fragmentada". La única verdad existente ha sido "la verdad científica", "la verdad objetiva", lo exacto, lo medible, "lo real", lo concreto. Aún y cuando, los límites de la realidad, son los límites de los métodos para percibir esa realidad. Las visiones del mundo y del desarrollo, solo ha sido posible verlas desde los cristales de esas perspectivas, solo dentro de los parámetros de su creación. Los caminos andados por aquellos países, las premisas de producción y productividad, la competitividad, el clientelismo y el consumo, los ingresos, la ciencia y el conocimiento han estado enfocadas al liberalismo económico desde finales del siglo XVIII, cuyas características se enfocaron a facilitar los procesos de acumulación (Carranza, 2013, p. 15).

La doctora Irma Becerra (2006 p. 71), plantea que "la polaridad que creíamos solo existía entre los países desarrollados por la hegemonía mundial, ha derivado ser una polaridad entre las clases dominantes de los países del tercer mundo, acostumbradas a inventar una visión de los vencidos". Mientras Carranza señala 
que gran parte del fracaso de los "Proyectos de Desarrollo del siglo XX" en regiones como el occidente de Honduras, se debe a que en los mismos han predominado visiones como estas; de carácter descendente, verticalistas y deterministas (Carranza, 2011. Op. cit., p. 75). En la academia o en las oportunidades de investigación que tienen los posgrados, poco estudiada ha sido la vasta mayoría de familias pobres socio económicamente excluidas que hoy cubren cerca del $83 \%$ de la población del país, donde la economía informal crece vertiginosamente como respuesta al desempleo, a la incertidumbre económica y a la inestabilidad. Además, una empresa privada que en un $95 \%$ la constituyen microempresas de carácter familiar, pequeñas y medianas, que hasta ahora son poco conocidas. Esto, generalmente no ha sido sujeto de estudio, análisis y valoraciones; mucho menos ser parte de los pensum de las carreras que en la educación formal se imparten. Como se estudian tan poco, es como que no estuvieran, es como dice la sabiduría popular "no mata la muerte, mata el olvido". Son poblaciones que estando tan cerca, están tan lejanas a la vez.

En cuanto al entorno de un centro universitario, no solo es el contexto físico en el cual está situado, no solo es la ubicación ni el espacio territorial de donde convergen los estudiantes, ni la parte del país de la cual se sirve; sino, es el contexto del cual la universidad vive y a la cual se debe. La academia está allí para servir, contribuyendo a su desarrollo, para estudiar, entender y comprender lo que allí pasa. El conocimiento científico universal, las premisas de los fundamentos teóricos y científicos que sustentan la praxis, el entendimiento local construido socialmente, la cultura como base del desarrollo, deberán ser componentes de nuevos sistemas sincréticos y abiertos del conocimiento. Porque solo al investigar, estudiar y profundizar, -en ese orden-, se podrán plantear alternativas y acelerar sus procesos, sus intereses y características económicas, sociales, ecológicas, estructurales, antropológicas, son esas "realidades" precisamente con las que hay que trabajar. Es necesario conocerlas, estudiarlas, identificarlas y sobre todo, comprenderlas y respetarlas. Es buscar planteamientos como los de Amartya Sen, Jeffrey Sachs, Manfred Max Neef, Roberto Sosa, Raúl Prebisch, Leonardo Boff, Ventura Ramos, Francisco Gutiérrez, Ramón Oquelí, Gautama Fonseca, Alberto Arroyo, Ignacio Medina, Gloria Lara, Helen Umaña, Julio Escoto, Jon Sobrino, entre otros, grandes pensadores, hombres y mujeres, que han amado, estudiado y servido a sus países y a esta América desigual.

Por lo tanto, los posgrados y su pertinencia en la investigación científica, deben comprender las realidades paralelas, la incertidumbre, el caos, la subjetividad y la cultura como fuente de conocimiento, de ciencia y desarrollo, la complejidad, la transdisciplinariedad y la interdisciplinariedad, la ciencia con conciencia, los 
enfoques sistemáticos que hasta hoy no han tenido parte en el camino hacia las nuevas ciencias, hacia nuevos paradigmas, todo esto, debe ser parte de una nueva visión y camino a construir. No se puede desarrollar algo sin conocerlo y sin conocer los contextos, como sus procesos de intercambio. No se trata de llevar caminos hechos como dice Morín, se trata de construirlos. La investigación en los posgrados, debe estudiar la conjugación de los procesos de producción, procesamiento y comercialización, la distribución de los ingresos y la dinámica de reinversión, así como comprender los subsistemas, actores y fuerzas que intervienen en la región. Ese mundo, es precisamente el que debe estudiarse desde los espacios académicos, desde la especialización. Eso sería entonces, acercarse a la construcción de nuevos caminos -hasta hoy negados-, hacia escenarios de otros niveles de desarrollo regional, local, humano, ambiental, endógeno, multiétnico, pluricultural, horizontales y menos hegemónicos.

En la búsqueda del conocimiento se trata de "Aprender a Aprender", donde participar sea más que contribuir a tomar decisiones o validar procesos, donde la palabra democracia tenga más significado y lo técnico sea una herramienta, no un fin. Los investigadores de nuestra universidad deben comprender que desde siempre y en diferentes tiempos, lugares e intensidades, la región de occidente ha funcionado como un sistema abierto y complejo. Al retomar estos elementos, los estudiantes de pregrado y posgrado desde diferentes perspectivas, producirían luces y elementos para una mejor comprensión. Es como dice Joseph Malta, "entender la realidad de la gente, buscarle el rostro humano". Porque no se puede plantear algo sin conocerlo, sin estudio; "sin amor, sin compromiso, sin interés ni reflexión, siempre seremos desconocidos, incluso para nosotros mismos; miraremos al espejo y con desenfado y desconfianza juzgaremos la figura que allí se refleja, conociéndonos, seremos extraños" (Carranza, 2013. Op. cit., p. 105).

Las universidades son una expresión a escala de las sociedades que les dan existencia, son vidas holográficas y complementarias. El quehacer de la universidad y su contexto, son vidas paralelas que coexisten y se alimentan mutuamente, muchas veces sin ni siquiera saberlo, conocerlo o estar conscientes de ello. En la investigación los pregrados deberían ser un inicio, por su proceso de formación y por sus aportes, donde comiencen estas aproximaciones. Los posgrados por su parte, deberán entonces ser los espacios para profundizar, para entrar a los tejidos de sociedades muy profundas, como las poblaciones rurales de esta parte del país, desde diferentes perspectivas; funcionalistas, estructuralistas, técnico-científicas, sostenibles y humanas.

Una región define sus posibilidades de vida en función de las articulaciones 
internas y externas en permanente ajuste y adaptación; en procesos siempre creativos y caóticos, amorfos, asimétricos y cambiantes. Hasta ahora en esta región se han realizado inversiones con diferentes enfoques, escuelas y tendencias, que en la mayoría de los casos no llegaron a sus destinatarios, pero justificaron la existencia de los proyectos. Hubo otros que sí contribuyeron a impulsar su desarrollo. Eso es la investigación; conocer y conocernos, ciencia con conciencia, búsqueda, encuentros y reencuentros. No se trata solamente de reproducir, es aprender y crecer. "Aprender es vivir" dice Humberto Maturana y añade "todo proceso de vida, es un proceso de aprender". Se trata de la búsqueda y la construcción de una nueva epistemología desde dentro, desde una visión incluyente y principalmente, con un irrestricto respeto a la condición humana.

\section{CONSIDERACIONES FINALES}

- La región del occidente de Honduras, es un vasto mundo de trabajos realizados y estudios a realizar. A pesar de sus precarias condiciones de vida, la región está llena de múltiples potencialidades y posibilidades de desarrollo. Los posgrados podrían ser espacios interesantes para entrar al conocimiento de las complejidades de esta región, donde se tenga la posibilidad de indagar, articular y construir saberes hacia la búsqueda de una nueva epistemología desde una visión incluyente y con rostro humano.

- El pensamiento descendente academicista que ha predominado en las universidades de Honduras y en cada uno de sus Centros Regionales, propias del paradigma reduccionista, positivista cartesiano, deberá abrir espacios hacia nuevos enfoques y conceptos que permitan acercarse a una mejor comprensión de esas realidades cambiantes en la sociedad.

- El contexto de los Centros Regionales Universitarios permite que de su estudio y conocimiento, puedan construirse propuestas de nuevas interpretaciones y procesos de cambios, hacia mejores realidades en las que podrían vivir las poblaciones hasta ahora desposeídas. Para ello, se deberán replantear y fortalecer el desempeño de sus docentes; pasando de una actividad exclusiva de la docencia, hacia áreas de investigación y vinculación con la sociedad.

- La deuda de la investigación y sus cuotas no satisfechas en la región, deberán ser temas de estudio y análisis permanente. La coordinación interinstitucional con las demás universidades, es un imperativo impostergable que la UNAH y sus centros regionales deberán asumir como un reto, esto permitirá acercarse más para comprender los problemas y la búsqueda de sus soluciones y cumplir de esta manera la misión que por mandato la nación nos ha encomendado. 


\section{AGRADECIMIENTOS}

Alos compañeros del equipo técnico de la Coordinación Regional de Investigación Científica CRIC. Al director del CUROC y a cada uno de nuestros colegas de trabajo.

\section{BIBLIOGRAFÍA}

Becerra, I. (2006). La América Encubierta. Honduras: Baktun

Barahona, M. (2002). Evolución Histórica de la Identidad Nacional (2da. ed.).

Tegucigalpa, Honduras: Editorial Guaymuras.

Bohn, D. (2001). Sobre El Dialogo. (2da. ed.). España: Kairós.

Carranza, R. (2013). Eco Antropología del Aprendizaje. Tesis doctoral no publicada. Universidad de La Salle, Costa Rica.

Carranza Ramos, A. (2012). Historia y Cultura del Café en el Occidente de Honduras. Aromas, Montañas y Gente. Tegucigalpa, Honduras: PROMECAFE/IHCAFE/BID/FOMIN. Impresiones Industriales.

Carranza, R. (2011). Antropología del Desarrollo. Tesis de maestría no publicada. Universidad Nacional Autónoma de Honduras. Santa Rosa de Copán, Honduras.

Constitución de la República. (2008). Título III, Capítulo VIII. Tegucigalpa, Honduras: Guaymuras.

Carranza Ramos, A. (2004). Palabras de Maíz y Barro. San Pedro Sula, Honduras: Diseños Impresos Múltiples DIM.

Castañeda, G. A. (1946). Una Ciudad de Honduras. La Sultana de Occidente. San Pedro Sula, Honduras: Biblioteca de la Sociedad de Geografía e Historia de Honduras.

Leyva, H. M. (1991). Documentos Coloniales de Honduras. Choluteca, Honduras: Colección Padre Manuel Subirana 3, Centro de Publicaciones Obispado de Choluteca.

Malta, J. (2013). Desarrollo para la Gente. Bases del Inclusionismo. Tegucigalpa, Honduras: Ideas Litográficas.

Morín, E. UCR (2010). El Método V. Cuarto Congreso sobre Complejidad, Universidad de Costa Rica.

Newson, L. (2000). El Costo de la Conquista. Tegucigalpa, Honduras: Editorial Guaymuras.

Orellana, J. (2010). San Miguel de Quezailica. Un Camino Hacia el Reencuentro. Tegucigalpa, Honduras: Guardabarranco.

PNUD (2009). Informe sobre Desarrollo Humano Honduras 2008/2009. San José, Costa Rica: Litografía e Imprenta LIL, S.A. 
Paz, O. (2008). El Laberinto de la Soledad. (3ra. ed.). México: Fondo de Cultura Económica.

PLATS-UNAH (2005). El Occidente de Honduras, tierra de carencias y esperanzas: tendencias de desarrollo e impactos de los procesos de intervención pública en los últimos 50 años. Tegucigalpa, Honduras: Alin Editora.

Rivas, R. D. (2004). Pueblos Indígenas y Garífunas de Honduras, (Una Caracterización). Tegucigalpa, Honduras: Editorial Guaymuras.

Varela, G. (2011). Historia de Honduras. Tegucigalpa, Honduras: Taller de Impresión Espinal Moncada.

Ventura, L. (2009). EI Linaje de Lara en Honduras, siglos XVI al XIX Conquistadores, encomenderos y hacendados. Tegucigalpa, Honduras: Instituto Hondureño de Antropología e Historia.

Zúñiga, A. (2000). Historia de Honduras. San Pedro Sula, Honduras: Impresos Comerciales Hernández. 